reduce professional isolation and stigma, enhance status and so improve recruitment. This may or may not be true, but I wonder about the attitude of neurologists to his proposal. The working life of a general adult psychiatrist is not easy and I think neurologists are likely to resist his advances. I don't know many who would be willing to regularly attend community-based mental health act assessments in inconvenient circumstances, subject themselves to crossexamination by enthusiastic lawyers in front of their patients at mental health tribunals, defend their practice at critical legalistic external inquiries, or subject themselves to the restrictions imposed by 'new ways of working'. Psychiatric practice certainly needs to be reformed but a more practical analysis of our problems is urgently required. In my opinion, our College must lead on these issues. If it continues to equivocate it will quickly become an irrelevance.

Keith E. Dudleston, Retired Consultant Psychiatrist, Ivybridge, UK, email: dudleston@btinternet.com

1 Fitzgerald M. Do psychiatry and neurology need a close partnership or a merger? BJPsych Bull 2015; 39: 105-7.

doi: $10.1192 / \mathrm{pb} .39 .5 .263 a$

\section{Can psychiatry and neurology 'simply' merge?}

I appreciate Professor Fitzgerald's citation of my 2005 article, titled 'Why psychiatry and neurology cannot simply merge', 1,2 however, he seems to have misconstrued the essential nature of my argument. He positions his discussion of my article just after the statement, 'The chorus of disapproval against neuropsychiatry has certainly grown'. But I would like to assure Professor Fitzgerald that I am not, nor have I ever been, part of such a 'chorus'. A careful reading of my article will show that the key word in my argument is 'simply'. I am not opposed in any way to integrating neurology and psychiatry; rather, I argue that certain types of 'bridging' concepts and constructs would be necessary to bring about such a union.

I describe neuropsychiatry as 'a vitally important transitional stage in the development of brain science'. Indeed, I would argue that neuropsychiatry is the crucible within which the discourses of psychiatry and neurology will eventually 'bond', producing a narrative that incorporates the dialectical and subtextual understanding of psychiatry into the framework of neurophysiology and neuropathology. But until such a meta-narrative has evolved, there cannot be a genuine merger of psychiatry and neurology. Or rather, we should say that without such a meta-narrative, the nature of the merger would be more like the grafting of an oak branch onto a maple tree than the hybridisation of two varieties of rose. ${ }^{2}$

I fully agree with Professor Fitzgerald that 'the separation of neurology from psychiatry has led to a separation of the brain from the mind - the physical from the mental - which has been unhelpful for both disciplines'. That said, I do not accept the view that psychiatric disease is best described as 'brain disease' or that mental constructs are 'reducible' to mere physiological or neuroanatomical terms. But this is a complicated philosophical issue best left for a longer communication. $^{3}$
Stated briefly, I believe that 'disease' is most usefully predicated of persons, not minds or brains, and that there are ways in which a union of neurology and psychiatry could contribute to a very rich understanding of the human person, and how personhood is undermined and compromised by disease states like schizophrenia. ${ }^{4}$

Ronald Pies, Professor of Psychiatry, SUNY Upstate Medical University, Syracuse, New York, and Tufts University School of Medicine, Boston, USA email: ronpies@massmed.org

1 Fitzgerald M. Do psychiatry and neurology need a close partnership or a merger? BJPsych Bull 2015; 39: 105-7.

2 Pies R. Why psychiatry and neurology cannot simply merge. J Neuropsychiatry Clin Neurosci 2005; 17: 304-9.

3 Pies R. Mind-language in the age of the brain: is "mental illness" a useful term? J Psychiatr Pract 2015; 21: 79-83.

4 Pies R. Trivializing the suffering of psychosis. Psychiatr Times 2014; 22 December.

doi: $10.1192 / p b .39 .5 .264$

\section{Fully inform the Martian}

At first glance, Reilly's thesis appears reasoned and structured. ${ }^{1}$ But his argument is flawed, such that he misses the most important reason for the distinction between psychiatry and neurology, with which a Martian would surely concur.

Reilly states that 'most organs (such as lungs, kidneys, hearts and eyes) are treated by a single medical specialty'. Not so. A cardiac surgeon operates on the heart, determines which patients would benefit from surgery, and manages pre- and post-operative care. A cardiologist's talents lie elsewhere.

Similarly, the division between psychiatry and neurology is defined by knowledge and skill. This is no artificial distinction imposed by a quirk of history, but reflects a difference in the very nature of the knowledge and skill base developed by doctors as they specialise. One cannot expect every trainee neurologist to additionally become expert in, say, holistic and developmental assessment, psychological formulation and complex diagnostic classifications of a nature unknown outside psychiatry. These are for trainee psychiatrists to focus on.

Doctors do not practise in isolation, but as members of multidisciplinary teams. Nurses and others develop similarly specialist knowledge and skills to work with patients with broadly different presentations.

Of course, there are small areas of overlap, but Reilly falsely dichotomises these to fuel his argument: I had no idea conversion disorder was the preserve of neurologists. At best, he puts forward a case for closer working and more shared care of patients between the two specialties. But two specialties they most assuredly are.

Richard Braithwaite, Consultant Psychiatrist, Isle of Wight NHS Trust, UK, email: richard.braithwaite@iow.nhs.uk

1 Reilly TJ. The neurology-psychiatry divide: a thought experiment. BJPsych Bull 2015; 39: 134-5.

doi: 10.1192/pb.39.5.264a 